\title{
Millimeter and Sub-millimeter Wave Response of Two- Dimensional Hot Electrons in double delta doped PbTe Quantum Well
}

\author{
Ankush Ghosh \\ Jadavpur \\ University \\ Kolkata 700032 \\ India
}

\author{
N Basanta Singh \\ Manipur Institute \\ of Technology \\ Imphal 795004 \\ India
}

\author{
Sanjoy Deb \\ Jadavpur \\ University \\ Kolkata 700032 \\ India
}

\author{
Goutham M A \\ Jadavpur \\ University \\ Kolkata 700032 \\ India
}

\author{
Subir Kumar \\ Sarkar \\ Jadavpur \\ University \\ Kolkata 700032 \\ India
}

\begin{abstract}
Small-signal ac transport of degenerate two-dimensional hot electrons in PbTe quantum wells is studied here incorporating deformation potential acoustic, polar optic and ionized background and remote impurity scatterings in the framework of heated drifted Fermi-Dirac distribution. The effects of double delta doping on millimeter and sub-millimeter wave response of two-dimensional hot electrons in PbTe quantum well is investigated. The inclusion of delta doping is found to enhance the two-dimensional electron density which in turn improves the ac mobility in the $\mathrm{PbTe}$ quantum wells thereby providing scope of getting higher speed devices in future.
\end{abstract}

\section{General Terms}

Quantum well nanostructure

\section{Keywords}

Quantum wells, delta doping, carrier scattering, polar optical phonon, coulomb interaction, sheet density.

\section{INTRODUCTION}

Advances in crystal growth techniques such as fine line lithography, molecular beam epitaxy (MBE) and Metal oxide chemical vapour deposition (MOCVD) have made possible the fabrication of quasi-low-dimensional structures such as Quantum wells (QWs). The possibility of realization of high speed nano devices using such structures has stimulated active research in quasi-low-dimensional structures.

$\mathrm{A} \mathrm{PbTe}$ quantum well is produced when a thin layer of $\mathrm{PbTe}$ is sandwiched between layers of higher band-gap semiconductor such as PbEuTe [1]. The growth of good quality $\mathrm{PbTe}$ quantum wells by MBE have already been reported in literatures $[2,3]$. Such structures are used in fabrication of infrared detectors and lasers. In $\mathrm{QW}$, the density-of-states and scattering rates of the carriers are different from those of bulk semiconductors. In such structures, mobility of the two-dimensional electron gas is enhanced considerably at low temperature because of modulation doping technique [4]. The carrier mobility in quantum wells can further be enhanced by placing an undoped spacer layer between the doped barrier and undoped channel layer [5]. The spacer layer increases the separation between the carriers and ionized donors thereby increasing the electron mobility because of less Coulomb interaction $[6,7]$.

The transport of charge carriers in a QW device depends on various factors like doping, carrier or sheet concentration, lattice temperature, applied field and carrier scattering etc. Due to structural limitations the maximum carrier density that can be obtained in conventional homogeneously doped QW is limited. This carrier density limit in QW can be surpassed in delta doped heterostructures [8].

The delta doping technique represents the ultimate technological limit of impurity profiles and this technique has resulted in a series of novel electronic and photonic devices $[9,10]$. When a semiconductor is delta doped, the ionized donor atoms create a continuously positive sheet of charge which bends the conduction band to form a V-shaped potential well [11]. When the dimension of the $\mathrm{V}$-shaped potential well is comparable to the de Broglie wavelength of free electrons, the electron energies for motion perpendicular to the delta doped plane are quantized into a number of discrete bands and a quasi-two dimensional electron gas (2DEG) is formed in the V-shaped potential well. The delta doped quantum wells have the following advantages. The first advantage arises from those of the delta-doping technique, including higher mobility, greater electron density and uniform electron distribution [12]. The second advantage is the reduction of size of the quantum wells [12]. Since the spatial distribution of dopants is well controlled and confined to a single atomic layer, the size of the quantum well is reduced.

It has been reported that the sheet density in delta doped structures is nearly doubled with respect to the densities obtained in modulation doped heterostructures [13,14]. However, most of the work in this area is concentrated in finding the carrier density and mobility in single sided delta doping heterostructures. From the available literature, it appears that there is still a dearth of research activities in the area of delta doped semiconductors. All these have motivated us to study the effects of double sided delta doping on the carrier concentration and the small-signal mobility of the two dimensional hot electrons in a PbTe quantum well. 
In the present work, we investigate the two dimensional electron densities confined in the quantum wells in delta doped $\mathrm{PbTe} / \mathrm{PbEuTe}$ quantum well structures and show that the $2 \mathrm{D}$ electron density can be enhanced due to delta doping in the barrier $\mathrm{PbEuTe}$ layer. We also investigate the ac mobility values in the delta doped PbTe quantum well in the framework of a heated drifted Fermi-Dirac distribution function and compared them with conventional homogeneously doped PbTe QW. The carrier scattering by longitudinal optic phonon, deformation potential acoustic phonons, remote and background-ionized impurities are incorporated in the present calculations.

\section{THEORY}

Let us consider a $\mathrm{PbTe} / \mathrm{PbEuTe}$ square $\mathrm{QW}$ of infinite barrier height. The PbEuTe barriers are symmetrically delta-doped at a distance Ls from the heterojunction interface. A schematic diagram and a simplified energy band diagram of the double delta doped quantum well are shown in Figs. 1 and 2.

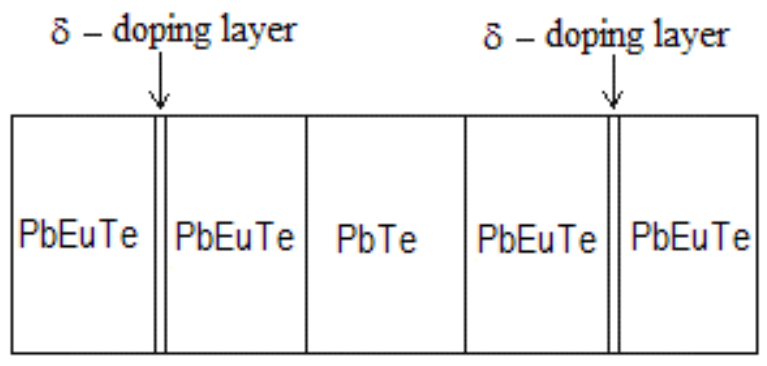

$\kappa \mathrm{Ls} \rightarrow \mathrm{Lz} \rightarrow \mathrm{Ls} \rightarrow$

Fig 1: A schematic diagram of a delta doped $\mathrm{PbTe} / \mathrm{PbEuTe}$ Quantum well

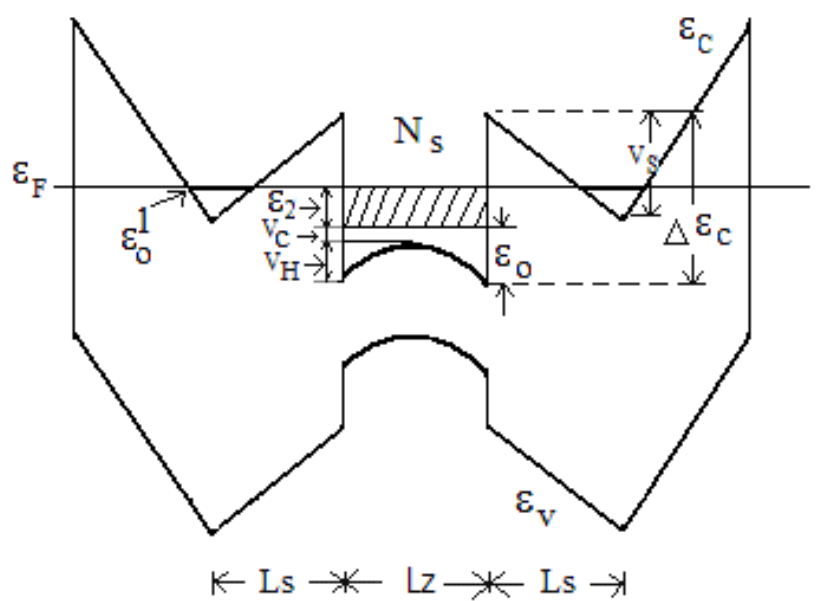

Fig 2: A simplified Energy band diagram of a delta doped PbTe/PbEuTe Quantum well

The energy gap of Pb1-xEuxTe is a function of $\mathrm{x}$ and is given by $\mathrm{Eg}(\mathrm{meV})=199+4480 \mathrm{x}$ [1]. For $\mathrm{x}=0.1$, assuming a band discontinuity $\Delta \mathrm{Ec}=0.55 \Delta \mathrm{Eg}$, the conduction band offset between the well and barrier layer is calculated as $246 \mathrm{meV}$. The 2D carrier concentration, QW width and other parameters used here are such that there is only one subband in the well and the carriers populate only the lowest subband. The zero order approximation for the electronic wave function and potential in the QW are given by [15].

$\psi_{o}(z)=\left(2 / L_{Z}\right)^{1 / 2} \operatorname{Cos}\left(\frac{\pi z}{L_{Z}}\right)$

and

$V_{o}(z)=-\frac{e^{2} N_{S}}{k_{1} L_{Z}}\left\{\left[1-\cos \left[\frac{2 \pi z}{L_{Z}}\right]\right] \frac{L_{z}^{2}}{4 \pi^{2}}+\frac{z^{2}}{2}\right\}$

The energy balance equation of the QW can be written as

$\Delta \varepsilon_{c}=V_{H}+V_{c}+\varepsilon_{2}+V_{s}-\varepsilon_{0}^{1}-\left(\varepsilon_{F}-\varepsilon_{0}^{1}\right)$

Assuming $\left(\varepsilon_{F}-\varepsilon_{0}^{1}\right)=0$, Eq. 3 reduces to

$\Delta \varepsilon_{c}=V_{H}+V_{c}+\varepsilon_{2}+V_{s}-\varepsilon_{0}^{1}$

The value of $V_{c}$ from the first-order perturbation theory is

$V_{C}=\frac{h^{2}}{8 m^{*} L_{Z}^{2}}-\frac{e^{2} N_{S} L_{Z}}{24 k_{1}}\left(1-\frac{3}{\pi^{2}}\right)$

The remaining terms of (4) are given as $[15,16]$

$$
\begin{aligned}
& V_{H}=\frac{e^{2} N_{S} L_{Z}}{8 k_{1}}\left(1+\frac{4}{\pi^{2}}\right) \\
& \varepsilon_{2}=\frac{\pi h^{2}}{m^{*}} N_{S} \\
& V_{S}=\frac{e^{2} N_{S}}{2 k_{2}} L_{Z}
\end{aligned}
$$

$$
\begin{aligned}
& \varepsilon_{n}^{1}=2^{-7 / 3}(n+1)^{2 / 3}\left(e^{2} 2 \pi \hbar n^{2 D} / k_{2} \sqrt{m^{*}}\right)^{2 / 3} \\
& \Delta \varepsilon_{c}=0.66 \Delta \varepsilon_{g}
\end{aligned}
$$

In the above expressions $\mathrm{k}_{1}$ and $\mathrm{k}_{2}$ are the dielectric constants in $\mathrm{PbEuTe}$ and $\mathrm{PbTe}$, respectively, $K_{B}$ is Boltzmann's constant, $\Delta \varepsilon_{\mathrm{g}}$ is change in band gap, $\mathrm{N}_{\mathrm{S}}$ is the carrier concentration in the well and $n^{2 D}$ is the donor density.

Inserting the above values in Eq. 4, we derived the expression for 2D electron density in the delta doped QW which is given as

$$
N_{S}=\frac{\left(\Delta \varepsilon_{C}-\frac{h^{2}}{8 m^{*} L_{Z}^{2}}+2^{-7 / 3}\left(e^{2} h n^{2 D} / k_{2} \sqrt{m^{*}}\right)^{2 / 3}\right)}{\left(\frac{e^{2} L_{Z}}{8 k_{1}}\left(1+\frac{4}{\pi^{2}}\right)-\frac{e^{2} L_{Z}}{24 k_{1}}\left(1-\frac{3}{\pi^{2}}\right)+\frac{\pi h^{2}}{m^{*}}+\frac{e^{2}}{2 k_{2}} L_{s}\right)}
$$

The 2D electron density for the homogeneously doped QW is derived using the procedure adopted in Ref. 15.

Reduced ionized impurity scattering and improved carrier concentration in the QW establish a strong electron-electron interaction. A heated drifted Fermi-Dirac distribution function for the carriers characterized by an electron temperature $T_{e}$, and a drifted crystal momentum $p_{d}$ [17] is hence assumed for the carriers. In the presence of an electric field $F$ applied parallel to 
the heterojunction interface, the carrier distribution function $f(\vec{k})$ is expressed as,

$f(\vec{k})=f_{o}(E)+\frac{\hbar \vec{p}_{d} \vec{k}}{m^{*}}\left(-\frac{\partial f_{o}}{\partial E}\right) \cos \gamma$

where, $\vec{k}$ is the two-dimensional wave vector associated with the kinetic energy E, $\mathrm{m} *$ is the electronic effective mass and $\gamma$ is the angle between the applied electric field $\vec{F}$ and the two dimensional wave vector $\vec{k}, f_{o}(E)$ is the Fermi-Dirac distribution function for the carriers, $\vec{p}_{d}$ is the drift crystal momentum and $\hbar$ is Planck's constant divided by 2 п.

Electron scattering by polar optic phonon, screened deformation potential acoustic phonon, background-ionized and remote impurities are considered in the calculations. Here we consider bulk-like phonon to avoid unmanageably complicated expression of scattering rates without greatly sacrificing the accuracy of the results [17]. The effects of screening are insignificant for polar optic phonons in the temperature range of interest here [17]. Therefore, screening is ignored for LO phonon scattering. However, the effects of screening are incorporated for for acoustic and ionized impurity scattering.

An electric field of magnitude $F_{1}$ and the angular frequency $\omega$ superimposed on a moderate dc bias field $F_{0}$ is assumed to act parallel to the heterojunction interface. The net field is thus given by:

$F=F_{o}+F_{1} \sin \omega t$

As the electron temperature and the drift momentum depend on the field and the scattering processes, they will also have similar components with the alternating ones generally differing in phase. Thus

$T_{e}=T_{o}+T_{1 r} \sin \omega t+T_{1 i} \cos \omega t$

$p_{d}=p_{o}+p_{1 r} \sin \omega t+p_{1 i} \cos \omega t$

Where, $T_{0}$ and $\mathrm{p}_{0}$ are the steady state parts, $T_{l r}$ and $p_{l r}$ are real and $T_{l i}$ and $p_{l i}$ are imaginary parts of $T_{e}$, and $p_{d}$ respectively.

The energy and momentum balance equations obeyed by the carrier are:

$e p_{d} F / m^{*}+\langle d E / d t\rangle_{s c a t}=\frac{d\langle E\rangle}{d t}$

and

$e F+\langle d p / d t\rangle_{s c a t}=\frac{d p_{d}}{d t}$

Where - $\langle d p / d t\rangle$ and $-\langle d E / d t\rangle$, represents, respectively, the average momentum and energy loss due to scatterings and $\langle E\rangle$ depicts the average energy of a carrier with charge e. In the present model the effects of delta doping is included in the energy and momentum loss calculations to give more accurate results

We insert Eqs. 14 and 15 in Eqs. 16 and 17, retain terms up to the linear in alternating components and equate the steady parts and the coefficients of $\sin \omega t$ and $\cos \omega t$ on the two sides of the resulting equations following the procedure adopted in [17]. For a given electric field $F_{o}$, we solve for $p_{o}$ and $T_{o}$. The dc mobility $\mu_{\mathrm{dc}}$ and ac mobility $\mu_{\mathrm{ac}}$ are then expressed as:

$\begin{aligned} \mu_{d c} & =\frac{p_{o}}{m^{*} F_{o}} \\ \mu_{a c} & =\frac{\sqrt{p_{1 r}^{2}+p_{1 i}^{2}}}{m^{*} F_{1}}\end{aligned}$

The phase lag $\phi$, the resulting alternating current lags behind the applied field is expressed as

$$
\phi=\tan ^{-1}\left(-\frac{p_{1 i}}{p_{1 r}}\right)
$$

\section{RESULTS AND DISCUSSION}

Numerical calculations are performed for delta doped square quantum well of width Lz with the parameters give in Table I.

Table 1. Material Parameters of PbTe

\begin{tabular}{|c|c|}
\hline Parameters & Values \\
\hline Electron effective mass $\mathrm{m}^{*}(\mathrm{Kg})$ & $0.058 \mathrm{~m}_{0}$ \\
\hline $\begin{array}{l}\text { Longitudinal elastic constant } \\
\mathrm{C}_{1}\left(\mathrm{~N} \cdot \mathrm{m}^{-2}\right)\end{array}$ & $\begin{array}{c}12.43 \times 10^{10}(\text { at } 77 \mathrm{~K}) \& \\
10.80 \times 10^{10}(\text { at } 300 \mathrm{~K})\end{array}$ \\
\hline Static dielectric constant, $\mathrm{K}_{\mathrm{s}}$ & $\begin{array}{l}428(77 \mathrm{~K}) \& \\
380(300 \mathrm{~K})\end{array}$ \\
\hline Optic dielectric constant, $\mathrm{K}_{\infty}$ & 37 \\
\hline $\begin{array}{l}\text { Background ionized impurity } \\
\text { concentration, } \mathrm{n}_{\mathrm{bi}}\left(\mathrm{m}^{-3}\right)\end{array}$ & $6 \times 10^{21}$ \\
\hline LO phonon energy & $13.6 \mathrm{meV}$ \\
\hline Lattice Constant a $(\AA)$ & 6.46 \\
\hline Mass density $\rho\left(\mathrm{g} / \mathrm{cm}^{3}\right)$ & 2.12 \\
\hline L O Phonon angular frequency & $2.064 \times 10^{13}$ \\
\hline Acoustic deformation potential & $24 \mathrm{eV}$ \\
\hline
\end{tabular}

The variation of $2 \mathrm{D}$ electron sheet density $\mathrm{N}_{\mathrm{s}}$ with quantum well width $\mathrm{L}_{\mathrm{z}}$ is shown in Fig. 3. The curve $a$ is for delta doped QW and it is plotted with $\mathrm{Ls}=5 \mathrm{~nm}$ and doping density, $\mathrm{n}^{2 \mathrm{D}}=1 \times 10^{16} \mathrm{~m}^{-}$

${ }^{2}$. Curve $b$ is for homogeneously doped QW and it is plotted with $\mathrm{Ls}=5 \mathrm{~nm}$ and doping density of $1 \times 10^{24} \mathrm{~m}^{-3}$. The $2 \mathrm{D}$ electron sheet density increases with increasing $\mathrm{L}_{Z}$ and it is found to be higher for delta doped QW than the homogeneously doped QW. The high concentration of two dimensional electron gas in delta doped QW is due to the quantum size effect in the delta doped region and spatial localization of donor impurities [16]. The nature of variation can be explained in a manner similar to the work reported in [15]. 


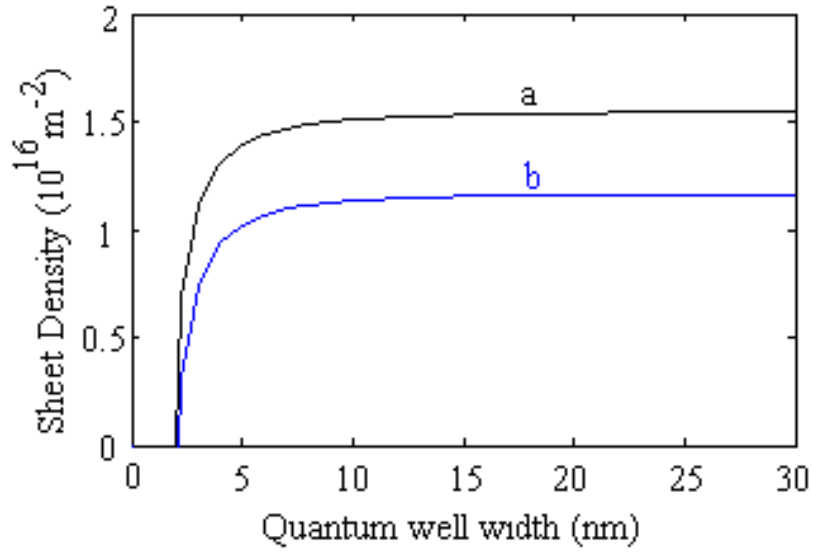

Fig 3: Variation of 2D electron density $\mathrm{N}_{\mathrm{S}}$ with quantum well width $L_{Z}$ for $x=0.3$ and $L s=5 \mathrm{~nm}$. Curve $a$ is for delta doped QW with a doping density of $1 \times 10^{16} \mathrm{~m}^{-2}$. Curve $b$ is for homogeneous doped $Q W$ with a doping density of $1 \times 10^{24} \mathrm{~m}^{-3}$

Fig. 4 shows the variation of ac mobility $\left(\mu_{\mathrm{ac}}\right)$ with frequency of the applied electric field for typical biasing field of $0.5 \times 105 \mathrm{~V} / \mathrm{m}$ at $77 \mathrm{~K}$. The curves are obtained with quantum well width $\mathrm{L}_{\mathrm{Z}}=10$ $\mathrm{nm}, \mathrm{L}_{\mathrm{S}}=5 \mathrm{~nm}$ and other parameters values same as those used in Fig. 3. The ac mobility is found to be higher for the delta doped QW than the homogeneous doped QW and it is due to more pronounced increase in ac mobility with enhanced carrier concentration in the former. The $3 \mathrm{~dB}$ cut-off frequency is 190 $\mathrm{GHz}$ for delta doping and $220 \mathrm{GHz}$ for homogeneous doping thereby reflecting that delta doped QWs are frequency limited although their performance is better within the operating frequency limit.

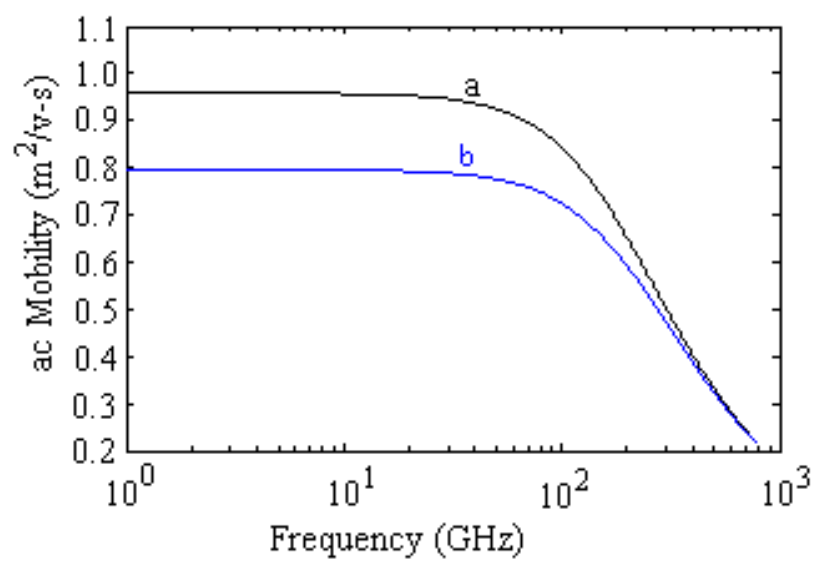

Fig 4: Variation of $\mu_{\mathrm{ac}}$ with frequency of the applied field at $77 \mathrm{~K}$ for $L_{\mathrm{Z}}=10 \mathrm{~nm}, \mathrm{~L}_{\mathrm{S}}=5 \mathrm{~nm}, \mathrm{~N}_{\mathrm{i}}=6 \times 10^{21} \mathrm{~m}^{-3}, \mathrm{n}^{\mathrm{D}}=1 \times 10^{24} \mathrm{~m}^{-3}$ and $\mathrm{n}^{2 \mathrm{D}}=1 \times 10^{16} \mathrm{~m}^{-2}$. The curves marked $a$ and $b$ represents the results for delta doped $\mathrm{QW}$ and conventional homogeneously doped $Q W$, respectively.

\section{CONCLUSION}

In this work we have investigated the effects of delta doping on the carrier concentration and ac mobility of the two dimensional hot electrons in a square $\mathrm{PbTe} / \mathrm{PbEuTe} \mathrm{QW}$. Our calculations show the basic effect of 2D electron concentration and mobility enhancement in $\mathrm{PbTe} \mathrm{QW}$ with delta doping in the barrier $\mathrm{PbEuTe}$ layer. The observed increase in $2 \mathrm{D}$ electron concentration of the PbTe quantum well in delta doped structures is due to quantum-size effect. The use of delta doping in the barrier layer leads to enhanced 2D electron density which in turn enhanced the carrier mobility in the QW thereby establishing the scope of getting high speed devices with delta doping. Our results will be best understood when they will appear in the literature as currently there is no such data available.

\section{ACKNOWLEDGMENTS}

Subir Kumar Sarkar thankfully acknowledges the financial sopprt obtained from DRDO

\section{REFERENCES}

[1] Chitta,V.A., Desrat,W., Maude, D.K., Piot, B.A., N.F., Oliveira Jr., Rappl, P.H.O., Ueta, A.Y., and Abramof, E. "Integer quantum Hall effect in a PbTe quantum well," Physica E, vol. 34, pp. 124-127, Apr. 2006.

[2] Koppensteiner, E. G., Springholz, P. Hamberger, and Bauer, G. " Molecular beam epitaxy of $\mathrm{PbTe} / \mathrm{EuTe}$ superlattices and their structural investigation by $\mathrm{x}$-ray diffraction using reciprocal space mapping," J. Appl. Phys., vol. 74, pp.6062-6071, Nov. 1993.

[3] Yuan, S., Springholz, G., Bauer, G., and Kriechbaum, M. “ Electronic and optical properties of $\mathrm{PbTe} / \mathrm{Pb}_{1-\mathrm{x}} \mathrm{Eu}_{\mathrm{x}} \mathrm{Te}$ multiple-quantum-well structures,“Phys. Rev. B. Vol. 49, pp. 5476-5489, Feb. 1994.

[4] Dingle, R., Stormer, H. L., Gossard A. C., and Wiegmann,W. "Electron mobilities in modulation-doped semiconductor heterojunction superlattices," Appl. Phys. Lett., vol. 33, no. 7, pp. 665-667, Oct. 1978.

[5] Harris, J. J., Pals, J. A. and Woltjer, R "Electronic transport in low-dimensional structures," Rep. Prog. Phys., vol. 52, pp.1217-1266, Oct. 1989.

[6] Foxon, C. T., Harris, J. J., Hilton, D., Hewett, J. and Roberts, C. "Optimisation of (Al,Ga)As/GaAs twodimensional electron gas structures for low carrier densities $\&$ ultra high mobilities at low temperature, "Semicond. Sci. Technol., vol. 4, pp. 582-595, Apr. 1989.

[7] Heiblum, M., Mendez, E. E. and Stern, F. "High mobility electron gas in selectively doped $n: \mathrm{AlGaAs} / \mathrm{GaAs}$ heterojunctions," Appl. Phys. Lett., vol. 44, no.11, pp. 1064-1066, Jun. 1984.

[8] Chico, L., Laskolski, W., Perez-Alvarez, R. and Garcia Moliner, F. "On the theory of GaAs-based quantum wells with external $\delta$-doping, J. Phys.: Condens. Matters, vol. 5, pp.9069-9076 Aug. 1993.

[9] Schubert, E. F., Cunningham, J. E. and Tsang, W.T. "Selfaligned enhancement-mode and depletion-mode GaAs field effect transistor employing the $\delta$-doping technique," Appl. Phys. Lett., 49, no. 25, pp. 1729-1731, Dec. 1986.

[10] Schubert, E. F., Fischer, A., Horikoshi, Y. and Ploog, K. "GaAs sawtooth superlattice laser emitting at wavelength 
$\lambda>0.9 \mu \mathrm{m}, "$ Appl. Phys. Lett., vol. 47, no. 3, pp. 219-221, Aug. 1985.

[11] Gossmann H.J. and Schubert, E.F. "Delta doping in Silicone," Solid State \& Material Sc., vol. 18, no. 1, pp. 167, Jan. 1993.

[12] Ikai, L., Chang, Y. C., Weng, H. M. and Chiang, J. C. "Two-dimensional electron gas in $\delta$-doped double quantum wells for photodetector application," J. Appl. Phys., vol. 81, no. 12, pp. 8112-8114, Jun. 1997.

[13] Cunningham, J. E., Tsang, W.T., Timp, G., Schubert, E. F., Chang, A. M. and Owusu-Sekyer, K. "Quantum size effects in monolayer doped heterostructures," Phys. Rev. B, vol. 37,no. 8, pp. 4317-4320, Mar. 1988.

[14] Kuo, T. Y., Cunningham, J. E., Schubert, E. F., Tsang, W. T., Chiu, T. H., Ren, R. and Fonstad, C. G. " Selectively $\delta$ doped quantum well transistor grown by gas source molecular beam epitaxy," J. Appl. Phys., vol. 64, no. 6, pp. 3324- 3327, Sept. 1988.

[15] Gomes, V.M.S., Chavas, A.S., Leite, J. R. and Worlock, J.M. "Self-consistent calculations of the two-dimensional electron density in modulation-doped superlattices," Phys. Rev. B, 35 (8), pp. 3984-3989, Mar. 1978

[16] Schubert, E. F., Cunningham, J. E., Tsang, W.T. and Timp, G.L. "Selectively $\delta$-doped $\mathrm{Al}_{\mathrm{x}} \mathrm{Ga}_{1-\mathrm{x}} \mathrm{As} / \mathrm{GaAs}$ heterostructures with high two-dimensional electron-gas concentrations $\mathrm{n}_{2 \mathrm{DEG}}>1.5 \times 10^{12} \mathrm{~cm}^{-2}$ for field-effect transistors, "Appl. Phys. Lett., vol. 51, no. 15, pp. 11701172 Oct. 1987.

[17] Sarkar, S. K., Ghosh, P. K. and Chattopadhyay, D. "Calculations of high-frequency response of twodimensional hot electrons in GaAs quantum wells," $J$. Appl. Phys., vol. 78, no. 1, pp. 283-287, July 1995. 